\title{
ON THE EXTENSION OF WHITNEY ULTRAJETS, II
}

\author{
ARMIN RAINER AND GERHARD SCHINDL
}

\begin{abstract}
We characterize the validity of the Whitney extension theorem in the ultradifferentiable Roumieu setting with controlled loss of regularity. Specifically, we show that in the main Theorem 1.3 of [16] condition (1.3) can be dropped. Moreover, we clarify some questions that remained open in [16].
\end{abstract}

\section{INTRODUCTION}

The main goal of this paper is to prove:

Theorem 1. Let $\omega$ be a non-quasianalytic concave weight function. Let $\sigma$ be a weight function satisfying $\sigma(t)=o(t)$ as $t \rightarrow \infty$. Then the following conditions are equivalent:

(i) For every compact $E \subseteq \mathbb{R}^{n}$ we have $j_{E}^{\infty}\left(\mathcal{B}^{\{\omega\}}\left(\mathbb{R}^{n}\right)\right) \supseteq \mathcal{B}^{\{\sigma\}}(E)$, where $j_{E}^{\infty}$ assigns to each $f \in C^{\infty}\left(\mathbb{R}^{n}\right)$ its infinite jet $\left(\left.f^{(\alpha)}\right|_{E}\right)_{\alpha \in \mathbb{N}^{n}}$ on $E$.

(ii) There is $C>0$ such that $\int_{1}^{\infty} \frac{\omega(t u)}{u^{2}} d u \leq C \sigma(t)+C$ for all $t>0$.

(Here $\mathcal{B}^{\{\omega\}}$ denotes the Roumieu class defined by the weight function $\omega$; we use the symbol $\mathcal{B}$ to emphasize that the defining estimates are global, cf. 16, 2.2 and 2.6].) It means that Theorem 1.3 of [16] holds without the assumption (1.3) that the associated weight matrix $\mathfrak{S}$ of $\sigma$ satisfies

$$
\forall S \in \mathfrak{S} \exists T \in \mathfrak{S} \exists C \geq 1 \forall 1 \leq j \leq k: \frac{S_{j}}{j S_{j-1}} \leq C \frac{T_{k}}{k T_{k-1}} .
$$

Theorem 1 is proved in Section 2. In Section 3 we clarify some questions that remained open in [16] and obtain several characterizations of concave weight functions. For an overview of the background of Theorem 1 we refer to the introduction in [16. We use the notation and the definitions of said paper; the concept of weight matrices is recalled in the appendix at the end of this paper.

Note that in the special case that $\omega$ and $\sigma$ coincide we recover the result of [1]:

Corollary 2. Let $\omega$ be a weight function. The following conditions are equivalent:

(i') For every compact $E \subseteq \mathbb{R}^{n}$ we have $j_{E}^{\infty}\left(\mathcal{B}^{\{\omega\}}\left(\mathbb{R}^{n}\right)\right)=\mathcal{B}^{\{\omega\}}(E)$.

(ii') There is $C>0$ such that $\int_{1}^{\infty} \frac{\omega(t u)}{u^{2}} d u \leq C \omega(t)+C$ for all $t>0$.

Date: November 13, 2018.

2010 Mathematics Subject Classification. 26E10, 30D60, 46E10, 58C25.

Key words and phrases. Whitney extension theorem in the ultradifferentiable setting, Roumieu type classes, controlled loss of regularity, properties of weight functions.

The first author was supported by the Austrian Science Fund (FWF) Project P 26735-N25. The second author was supported by FWF-Project J 3948-N35; within this project, he is an external researcher at the Universidad de Valladolid (Spain) for the period October 2016 - September 2018. 
Indeed, if $\omega$ satisfies (ii') then it is non-quasianalytic, equivalent to a concave weight function [9, Proposition 1.3], and $\omega(t)=o(t)$ as $t \rightarrow \infty$ [2, Remark 3.20]. That (ii') is a necessary condition for (i') is well-known. Note that also (i') implies that $\omega$ is non-quasianalytic. Indeed, if $\omega$ is quasianalytic, then the Borel map $j_{\{0\}}^{\infty}: \mathcal{B}^{\{\omega\}}\left(\mathbb{R}^{n}\right) \rightarrow \mathcal{B}^{\{\omega\}}(\{0\})$ is never surjective. For $t \neq O(\omega(t))$ as $t \rightarrow \infty$ this follows from [15], for $t=O(\omega(t))$ as $t \rightarrow \infty$ consider e.g. the formal series $\sum_{k=0}^{\infty} x^{k}$ which converges to the unbounded real analytic function $1 /(1-x)$ function for $|x|<1$.

\section{Proof of Theorem 1}

Preparations. First we recall a few definitions and facts. Let $m=\left(m_{k}\right)$ be a positive sequence satisfying $m_{0}=1$ and $m_{k}^{1 / k} \rightarrow \infty$. The log-convex minorant of $m$ is given by

$$
\underline{m}_{k}:=\sup _{t>0} \frac{t^{k}}{\exp \left(\omega_{m}(t)\right)}, \quad k \in \mathbb{N}
$$

where

$$
\omega_{m}(t):=\sup _{k \in \mathbb{N}} \log \left(\frac{t^{k}}{m_{k}}\right), \quad t>0 .
$$

The function $\omega_{m}$ is increasing, convex in $\log t$, and zero for sufficiently small $t>0$. Related is the function $h_{m}(t):=\inf _{k \in \mathbb{N}} m_{k} t^{k}$, for $t>0$, and $h_{m}(0):=0$. It is increasing, continuous, positive for $t>0$, and equals 1 for large $t$.

Let $m=\left(m_{k}\right)$ be a positive log-convex sequence (i.e., $m=\underline{m}$ ) such that $m_{0}=1$ and $m_{k}^{1 / k} \rightarrow \infty$. Then the functions $\bar{\Gamma}_{m}$ and $\underline{\Gamma}_{m}$ defined in [16, Definition 3.1] coincide, we simply write $\Gamma_{m}$ in this case; note that log-convexity and $m_{k}^{1 / k} \rightarrow \infty$ imply $m_{k} / m_{k-1} \rightarrow \infty$. Thus

$$
\Gamma_{m}(t)=\min \left\{k: h_{m}(t)=m_{k} t^{k}\right\}=\min \left\{k: \frac{m_{k+1}}{m_{k}} \geq \frac{1}{t}\right\}, \quad t>0 .
$$

By [16, Lemma 3.2], $\Gamma_{m}$ is decreasing, tending to $\infty$ as $t \rightarrow 0$, and

$$
k \mapsto m_{k} t^{k} \text { is decreasing for } k \leq \Gamma_{m}(t) .
$$

Recall that with every weight function $\sigma$ (always understood as defined in [16. Section 2.1]) is associated a weight matrix $\mathfrak{S}=\left\{S^{\xi}\right\}_{\xi>0}$, where

$$
S_{k}^{\xi}:=\exp \left(\frac{1}{\xi} \varphi^{*}(\xi k)\right), \quad \text { (here } \varphi=\sigma \circ \exp \text { and } \varphi^{*} \text { is its Young conjugate), }
$$

such that $\mathcal{B}^{\{\sigma\}}=\mathcal{B}^{\{\mathfrak{S}\}}$ and $\mathcal{B}^{(\sigma)}=\mathcal{B}^{(\mathfrak{S})}$ algebraically and topologically; cf. [16, 2.5] and [12]. In the following we set $s_{k}^{\xi}:=S_{k}^{\xi} / k !$.

The next proposition shows that for a weight function $\sigma$ which is equivalent to a concave weight function and satisfies $\sigma(t)=o(t)$ as $t \rightarrow \infty$ we additionally have $\mathcal{B}^{\{\sigma\}}=\mathcal{B}^{\{\underline{\mathfrak{S}}\}}$ and $\mathcal{B}^{(\sigma)}=\mathcal{B}^{(\underline{\mathfrak{S}})}$, where $\underline{\mathfrak{S}}=\left\{\underline{S}^{\xi}\right\}_{\xi>0}$ and

$$
\underline{S}_{k}^{\xi}:=k ! \underline{s}_{k}^{\xi} \text {. }
$$

In particular, $\underline{\mathfrak{S}}$ satisfies (11). We say that $\underline{S}^{\xi}$ is strongly log-convex meaning that $\underline{s}_{k}^{\xi}=\underline{S}_{k}^{\xi} / k$ ! is log-convex. (Note the abuse of notation: $\underline{S}^{\xi}$ is not necessarily the log-convex minorant of $S^{\xi}$; this will cause no confusion.) Recall that two weight functions $\omega$ and $\sigma$ are called equivalent if $\omega(t)=O(\sigma(t))$ and $\sigma(t)=O(\omega(t))$ as $t \rightarrow \infty$; this means that they define the same ultradifferentiable class. 
Proposition 3. Let $\sigma$ be a weight function satisfying $\sigma(t)=o(t)$ as $t \rightarrow \infty$ which is equivalent to a concave weight function. For each $\xi>0$ there exist constants $A, B, C>0$ such that

$$
A^{-1} s_{k}^{\xi / B} \leq \underline{s}_{k}^{\xi} \leq s_{k}^{\xi} \leq C^{k} \underline{s}_{k}^{B \xi} \quad \text { for all } k \in \mathbb{N} .
$$

Moreover, there is a constant $H \geq 1$ such that $\underline{s}_{j+k}^{\xi} \leq H^{j+k} \underline{s}_{j}^{2 \xi} \underline{s}_{k}^{2 \xi}$, for all $\xi>0$ and all $j, k \in \mathbb{N}$, and thus $h_{\underline{s}^{\xi}}(t) \leq h_{\underline{s}^{2 \xi}}(H t)^{2}$, for all $\xi>0$ and all $t>0$.

Proof. Clearly, $\underline{s}^{\xi} \leq s^{\xi}$. Let $\underline{S}_{k}^{\xi}:=k ! \underline{s}_{k}^{\xi}$. By [6, Lemma 3.6], $\omega_{S} \xi$ and $\omega_{\underline{S}} \xi$ are equivalent, in particular, there exists $C \geq 1$ such that

$$
\omega_{\underline{S}^{\xi}} \leq C \omega_{S^{\xi}}+C .
$$

By [16, Lemma 2.4(3)] and [14, Remark 2.5], we have

$$
2 \omega_{S^{2 \xi}} \leq \omega_{S^{\xi}}, \quad \text { for all } \xi>0 .
$$

If $n$ is an integer such that $B:=2^{n} \geq C$, then $\omega_{\underline{S}^{\xi}} \leq \omega_{S^{\xi} / B}+C$ and hence

$$
\underline{S}_{k}^{\xi}=\sup _{t>0} \frac{t^{k}}{\exp \left(\omega_{\underline{S}^{\xi}}(t)\right)} \geq e^{-C} \sup _{t>0} \frac{t^{k}}{\exp \left(\omega_{S^{\xi} / B}(t)\right)}=e^{-C} S_{k}^{\xi / B} .
$$

This shows the first inequality in (4).

By [16, Lemma 3.13], there exists $D \geq 1$ such that for all $\xi>0$,

$$
2 \omega_{s^{2 \xi}}(t) \leq \omega_{s \xi}(D t), \quad \text { for } t>0
$$

and therefore

$$
\underline{s}_{2 k}^{\xi}=\sup _{t>0} \frac{(D t)^{2 k}}{\exp \left(\omega_{s^{\xi}}(D t)\right)} \leq D^{2 k} \sup _{t>0} \frac{t^{2 k}}{\exp \left(2 \omega_{s^{2 \xi}}(t)\right)}=D^{2 k}\left(\underline{s}_{k}^{2 \xi}\right)^{2} .
$$

Thus, by [17, Theorem 9.5.1] (which is a generalization of [8]), there exists a constant $H \geq 1$ such that $\underline{s}_{j+k}^{\xi} \leq H^{j+k} \underline{s}_{j}^{2 \xi} \underline{s}_{k}^{2 \xi}$, for all $j, k$. That $h_{\underline{s}}^{\xi}(t) \leq h_{\underline{s}^{2} \xi}(H t)^{2}$, for all $\xi>0$ and all $t>0$, follows from [16. Lemma 3.12]. By [18, Proposition 3.6],

$$
2 \omega_{\underline{S}^{2 \xi}}(t) \leq \omega_{\underline{S}^{\xi}}(H t), \quad \text { for } t>0,
$$

for some (possibly different) $H \geq 1$. As above, using (15), we find $\omega_{\underline{S}^{B \xi}}(b t) \leq$ $\omega_{S \xi}(t)+1$ for some constant $0<b \leq 1$. Then

$$
\underline{S}_{k}^{B \xi}=\sup _{t>0} \frac{(b t)^{k}}{\exp \left(\omega_{\underline{S}^{B \xi}}(b t)\right)} \geq e^{-1} b^{k} \sup _{t>0} \frac{t^{k}}{\exp \left(\omega_{S^{\xi}}(t)\right)}=e^{-1} b^{k} S_{k}^{\xi} .
$$

The last inequality of (4) follows.

Proposition 3 alone is not enough to get rid of the assumption (11). It is not clear that $\underline{\mathfrak{S}}$ has the property that for all $S \in \underline{\mathfrak{S}}$ there is a $T \in \underline{\mathfrak{S}}$ such that $S_{2 k} / S_{2 k-1} \lesssim T_{k} / T_{k-1}$. Note that $\mathfrak{S}$ has this property (see [16, Lemma 2.4(4)]) and it enters crucially in Lemma 3.4 and Proposition 3.7 of [16].

We deal with this problem by introducing another intimately related weight matrix $\mathfrak{V}:=\left\{V^{\xi}\right\}_{\xi>0}$. For each $\xi>0$ we define $V_{k}^{\xi}:=k ! v_{k}^{\xi}$ by setting

$$
v_{k}^{\xi}:=\min _{0 \leq j \leq k} \underline{s}_{j}^{2 \xi} \underline{s}_{k-j}^{2 \xi}, \quad k \in \mathbb{N} .
$$


That means that for the sequence of quotients $v_{k}^{\xi} / v_{k-1}^{\xi}$ we have (cf. [7, Lemma 3.5])

$$
\left(\frac{v_{1}^{\xi}}{v_{0}^{\xi}}, \frac{v_{2}^{\xi}}{v_{1}^{\xi}}, \frac{v_{3}^{\xi}}{v_{2}^{\xi}}, \frac{v_{4}^{\xi}}{v_{3}^{\xi}}, \ldots\right)=\left(\frac{\underline{s}_{1}^{2 \xi}}{\underline{s}_{0}^{2 \xi}}, \frac{\underline{s}_{1}^{2 \xi}}{\underline{s}_{0}^{2 \xi}}, \frac{\underline{s}_{2}^{2 \xi}}{\underline{s}_{1}^{2 \xi}}, \frac{\underline{s}_{2}^{2 \xi}}{\underline{s}_{1}^{2 \xi}}, \frac{\underline{s}_{3}^{2 \xi}}{\underline{s}_{2}^{2 \xi}}, \frac{\underline{s}_{3}^{2 \xi}}{\underline{s}_{2}^{2 \xi}}, \ldots\right) \text {. }
$$

Thus the sequence $v^{\xi}=\left(v_{k}^{\xi}\right)$ is log-convex and satisfies

$$
\frac{v_{2 k-1}^{\xi}}{v_{2 k-2}^{\xi}}=\frac{v_{2 k}^{\xi}}{v_{2 k-1}^{\xi}}=\frac{\underline{s}_{k}^{2 \xi}}{\underline{s}_{k-1}^{2 \xi}}, \quad \text { for all } k \geq 1
$$

So, in view of (2),

$$
2 \Gamma_{\underline{s}^{2 \xi}}(t)=\Gamma_{v^{\xi}}(t), \quad \text { for all } t>0 .
$$

By Proposition 3, there is $H \geq 1$ such that for all $\xi>0$

$$
\underline{s}_{k}^{\xi} \leq H^{k} v_{k}^{\xi} \leq H^{k} \underline{s}_{k}^{2 \xi}, \quad \text { for all } k \in \mathbb{N} .
$$

Thus, we also have $\mathcal{B}^{\{\sigma\}}=\mathcal{B}^{\{\mathfrak{V}\}}$ and $\mathcal{B}^{(\sigma)}=\mathcal{B}^{(\mathfrak{V})}$ algebraically and topologically.

Proof of Theorem 1. The implication (i) $\Rightarrow$ (ii) follows from [2. So we only prove the converse implication. Condition (ii) means that the weight function

$$
\kappa(t):=\int_{1}^{\infty} \frac{\omega(t u)}{u^{2}} d u
$$

satisfies $\kappa(t)=O(\sigma(t))$ as $t \rightarrow \infty$, i.e., $\mathcal{B}^{\{\sigma\}} \subseteq \mathcal{B}^{\{\kappa\}}$. Now $\kappa$ is concave and $\kappa(t)=o(t)$ as $t \rightarrow \infty$, see [9. Proposition 1.3]. We will show that Whitney ultrajets of class $\mathcal{B}^{\{\kappa\}}$ admit extensions of class $\mathcal{B}^{\{\omega\}}$. Thus from now on we assume without loss of generality that $\sigma=\kappa$ is concave. Since $\omega$ is increasing we have $\sigma=\kappa \geq \omega$ and hence, if $\mathfrak{W}=\left\{W^{\xi}\right\}_{\xi>0}$ denotes the weight matrix associated with $\omega$,

$$
\underline{S}^{\xi} \leq S^{\xi} \leq W^{\xi}, \quad \text { for all } \xi>0 .
$$

Moreover, Proposition 3 as well as (8) and (9) apply. Let us now indicate the necessary changes in the proof of [16, Theorem 1.3]. The changes also lead to some simplifications. We provide details in the hope that this contributes to a better understanding.

- Every Whitney ultrajet $F=\left(F^{\alpha}\right)$ of class $\mathcal{B}^{\{\sigma\}}$ on the compact set $E \subseteq \mathbb{R}^{n}$ is an element of $\mathcal{B}^{\left\{V^{\xi}\right\}}(E)$ for some $\xi>0$, i.e., there exist $C>0$ and $\rho \geq 1$ such that

$$
\begin{gathered}
\left|F^{\alpha}(a)\right| \leq C \rho^{|\alpha|} V_{|\alpha|}^{\xi}, \quad \alpha \in \mathbb{N}^{n}, a \in E, \\
\left|\left(R_{a}^{k} F\right)^{\alpha}(b)\right| \leq C \rho^{k+1}|\alpha| ! v_{k+1}^{\xi}|b-a|^{k+1-|\alpha|}, \quad k \in \mathbb{N},|\alpha| \leq k, a, b \in E .
\end{gathered}
$$

Let $p \in \mathbb{N}$ be fixed (and to be specified later). Let $\left\{\varphi_{i, p}\right\}_{i \in \mathbb{N}}$ be the partition of unity provided by [16, Proposition 4.9], relative to the family of cubes $\left\{Q_{i}\right\}_{i \in \mathbb{N}}$ from [16. Lemma 4.7], and let $r_{0}=r_{0}(p)$ be the constant appearing in this proposition. The center of $Q_{i}$ is denoted by $x_{i}$. We claim that an extension of class $\mathcal{B}^{\{\omega\}}$ of $F$ to a suitable neighborhood of $E$ in $\mathbb{R}^{n}$ is provided by

$$
f(x):= \begin{cases}\sum_{i \in \mathbb{N}} \varphi_{i, p}(x) T_{\hat{x}_{i}}^{p\left(x_{i}\right)} F(x), & \text { if } x \in \mathbb{R}^{n} \backslash E, \\ F^{0}(x), & \text { if } x \in E,\end{cases}
$$

where, given $x \in \mathbb{R}^{n} \backslash E, \hat{x}$ is any point in $E$ with $d(x):=d(x, E)=|x-\hat{x}|$ and

$$
p(x):=\max \left\{2 \Gamma_{\underline{s}^{2 \xi}}(L d(x))-1,0\right\} .
$$


Here $L$ is a positive constant to be specified below. Recall that $Q_{i}^{*}$ is the closed cube with the same center as $Q_{i}$ expanded by the factor 9/8. By [16, Corollary $4.8]$,

$$
\frac{1}{2} d(x) \leq d\left(x_{i}\right) \leq 3 d(x), \quad \text { for all } x \in Q_{i}^{*} .
$$

Then $d(x)<1 /\left(3 L \underline{s}_{1}^{2 \xi}\right)$ guarantees that both $\Gamma_{\underline{s}^{2} \xi}\left(L d\left(x_{i}\right)\right)$ and $\Gamma_{\underline{s}^{2 \xi}}(L d(x))$ are $\geq 1$, by (2), thus $p\left(x_{i}\right)=2 \Gamma_{\underline{s}^{2 \xi}}\left(L d\left(x_{i}\right)\right)-1$ and $p(x)=2 \Gamma_{\underline{s}^{2 \xi}}(L d(x))-1$.

- Replace [16, Lemma 5.2] by the following lemma. The only difference in the proof is that one uses (8) instead of [16, (5.4)].

Lemma 4. There is a constant $C_{0}=C_{0}(n)>1$ such that, for all Whitney ultrajets $F=\left(F^{\alpha}\right)_{\alpha}$ of class $\mathcal{B}^{\left\{V^{\xi}\right\}}$ that satisfy (12) and (13), all $L \geq C_{0} \rho$, all $x \in \mathbb{R}^{n}$, and all $\alpha \in \mathbb{N}^{n}$,

$$
\left|\left(T_{\hat{x}}^{p(x)} F\right)^{(\alpha)}(x)\right| \leq C(2 L)^{|\alpha|+1} V_{|\alpha|}^{\xi}
$$

and, if $|\alpha|<p(x)$,

$$
\left|\left(T_{\hat{x}}^{p(x)} F\right)^{(\alpha)}(x)-F^{\alpha}(\hat{x})\right| \leq C(2 L)^{|\alpha|+1}|\alpha| ! v_{|\alpha|+1}^{\xi} d(x) .
$$

We remark that (here and below) by $\left(T_{\hat{x}}^{p(x)} F\right)^{(\alpha)}(x)$ we mean the $\alpha$-th partial derivative of the polynomial $y \mapsto T_{\hat{x}}^{p(x)} F(y)$ evaluated at $y=x$.

- Replace [16, Lemma 5.3] by:

Lemma 5. There is a constant $C_{1}=C_{1}(n)>0$ such that for all $L>C_{1} \rho$, all $\beta \in \mathbb{N}^{n}$, and all $x \in Q_{i}^{*}$ with $d(x)<1 /\left(3 L \underline{s}_{1}^{2 \xi}\right)$,

$$
\left|\partial^{\beta}\left(T_{\hat{x}_{i}}^{p\left(x_{i}\right)} F-T_{\hat{x}}^{\left.p\left(x_{i}\right)\right)} F\right)(x)\right| \leq C L^{|\beta|+1} \underline{S}_{|\beta|}^{2 \xi} h_{\underline{s}^{2 \xi}}\left(L d\left(x_{i}\right)\right) .
$$

Proof. It suffices to consider $|\beta| \leq p\left(x_{i}\right)=2 \Gamma_{\underline{s}^{2 \xi}}\left(\operatorname{Ld}\left(x_{i}\right)\right)-1=: 2 q-1$. Let $H_{1}$ denote the left-hand side of (17). By [16, Lemma 5.1 and Corollary 4.8] and (6),

$$
H_{1} \leq C\left(2 n^{2} \rho\right)^{2 q}|\beta| ! v_{2 q}^{\xi}\left(6 d\left(x_{i}\right)\right)^{2 q-|\beta|} \leq C\left(2 n^{2} \rho\right)^{2 q}|\beta| !\left(\underline{s}_{q}^{2 \xi}\right)^{2}\left(6 d\left(x_{i}\right)\right)^{2 q-|\beta|} .
$$

By the definition of $q, h_{\underline{s}^{2 \xi}}\left(L d\left(x_{i}\right)\right)=\underline{s}_{q}^{2 \xi}\left(L d\left(x_{i}\right)\right)^{q} \leq \underline{s}_{k}^{2 \xi}\left(L d\left(x_{i}\right)\right)^{k}$ for all $k$. Thus

$$
H_{1} \leq C\left(\frac{12 n^{2} \rho}{L}\right)^{2 q} L^{|\beta|}|\beta| ! \underline{s}_{|\beta|}^{2 \xi} h_{\underline{s}^{2 \xi}}\left(L d\left(x_{i}\right)\right) .
$$

If $L>12 n^{2} \rho$, then (17) follows.

- Replace [16, Lemma 5.4] by:

Lemma 6. There is a constant $C_{2}=C_{2}(n)>0$ such that for all $L>C_{2} \rho$, all $\beta \in \mathbb{N}^{n}$, and all $x \in Q_{i}^{*}$ with $d(x)<1 /\left(3 L \underline{s}_{1}^{2 \xi}\right)$,

$$
\left|\partial^{\beta}\left(T_{\hat{x}}^{p\left(x_{i}\right)} F-T_{\hat{x}}^{p(x)} F\right)(x)\right| \leq C\left(\frac{3 L}{n}\right)^{|\beta|+1} \underline{S}_{|\beta|}^{2 \xi} h^{2 \xi}(3 L d(x)) .
$$

Proof. Both $p\left(x_{i}\right)$ and $p(x)$ are majorized by $\Gamma_{v^{\xi}}(\operatorname{Ld}(x) / 2)$, indeed, by (8), (14), and since $\Gamma_{v^{\xi}}$ is decreasing,

$$
p\left(x_{i}\right)=2 \Gamma_{\underline{s}^{2 \xi}}\left(L d\left(x_{i}\right)\right)-1 \leq 2 \Gamma_{\underline{s}^{2 \xi}}\left(L d\left(x_{i}\right)\right)=\Gamma_{v^{\xi}}\left(L d\left(x_{i}\right)\right) \leq \Gamma_{v^{\xi}}(L d(x) / 2) .
$$

So the degree of the polynomial $T_{\hat{x}}^{p\left(x_{i}\right)} F-T_{\hat{x}}^{p(x)} F$ is at most $\Gamma_{v^{\xi}}(\operatorname{Ld}(x) / 2)$. The valuation of the polynomial is equal to $\min \left\{p\left(x_{i}\right), p(x)\right\}+1$ (unless $p\left(x_{i}\right)=p(x)$ in 
which case (18) is trivial) and so at least $2 \Gamma_{\underline{s}^{2} \xi}(3 L d(x))=: 2 q$, by (14). So if $H_{2}$ denotes the left-hand side of (18), then (see the calculation in [16, (5.7)])

$$
H_{2} \leq \frac{C|\beta| !}{(n d(x))^{|\beta|}} \sum_{j=2 q}^{\Gamma_{v} \xi(L d(x) / 2)}\left(2 n^{2} \rho d(x)\right)^{j} v_{j}^{\xi} .
$$

By (3), $v_{j}^{\xi}(\operatorname{Ld}(x) / 2)^{j} \leq v_{2 q}^{\xi}(\operatorname{Ld}(x) / 2)^{2 q}$ for $2 q \leq j \leq \Gamma_{v^{\xi}}(\operatorname{Ld}(x) / 2)$. By the definition of $q, h_{\underline{s}^{2 \xi}}(3 L d(x))=\underline{s}_{q}^{2 \xi}(3 L d(x))^{q} \leq \underline{s}_{k}^{2 \xi}(3 L d(x))^{k}$ for all $k$. With (6) this leads to

$$
\begin{aligned}
H_{2} & \leq \frac{C|\beta| !}{(n d(x))^{|\beta|}} \sum_{j=2 q}^{\Gamma_{v} \xi(L d(x) / 2)}\left(\frac{4 n^{2} \rho}{L}\right)^{j} v_{2 q}^{\xi}\left(\frac{L d(x)}{2}\right)^{2 q} \\
& \leq \frac{C|\beta| !}{(n d(x))^{|\beta|}} \sum_{j=2 q}^{\Gamma_{v} \xi(L d(x) / 2)}\left(\frac{4 n^{2} \rho}{L}\right)^{j}\left(\underline{s}_{q}^{2 \xi}\right)^{2}\left(\frac{L d(x)}{2}\right)^{2 q} \\
& \leq C\left(\frac{3 L}{n}\right)^{|\beta|}|\beta| ! \underline{s}_{|\beta|}^{2 \xi} h_{\underline{s}^{2 \xi}}(3 L d(x)) \sum_{j=2 q}^{\Gamma_{v} \xi}\left(\frac{L d(x) / 2)}{L}\right)^{j} .
\end{aligned}
$$

If we choose $L \geq 8 n^{2} \rho$, then the sum is bounded by 2 , and (18) follows.

- Assume that $L$ is chosen such that

$$
L>\max \left\{C_{0}, C_{1}, C_{2}\right\} \rho
$$

so that (15), (16), (17), and (18) are valid. Recall that $\mathfrak{W}$ denotes the weight matrix associated with $\omega$. The next lemma is a substitute for the claim in the proof of Theorem 5.5 in 16 .

Lemma 7. There exist constants $K_{j}=K_{j}(n, \omega), j=1,2,3$, such that the following holds. If $p=K_{1} L$ and $L>K_{2} \rho$, then there exist a weight sequence $W \in \mathfrak{W}$ and a constant $M_{1}=M_{1}(n, \omega, L)>0$ such that for all $x \in \mathbb{R}^{n} \backslash E$ with $d(x)<$ $\min \left\{r_{0} /\left(3 B_{1}\right), 1 /\left(3 L \underline{s}_{1}^{2 \xi}\right)\right\}$ and all $\alpha \in \mathbb{N}^{n}$,

$$
\left|\partial^{\alpha}\left(f-T_{\hat{x}}^{p(x)} F\right)(x)\right| \leq C M_{1}^{|\alpha|+1} W_{|\alpha|} h_{\underline{s}^{4 \xi}}\left(K_{3} L d(x)\right),
$$

where $C$ and $\rho$ are the constants from (12) and (13) (and $B_{1}$ is the universal constant from [16, Lemma 4.7]).

Proof. By the Leibniz rule,

$$
\partial^{\alpha}\left(f-T_{\hat{x}}^{p(x)} F\right)(x)=\sum_{\beta \leq \alpha}\left(\begin{array}{c}
\alpha \\
\beta
\end{array}\right) \sum_{i} \varphi_{i, p}^{(\alpha-\beta)}(x) \partial^{\beta}\left(T_{\hat{x}_{i}}^{p\left(x_{i}\right)} F-T_{\hat{x}}^{p(x)} F\right)(x) .
$$

Now (17) and (18) imply, that for $x \in Q_{i}^{*}$ with $d(x)<1 /\left(3 L \underline{s}_{1}^{2 \xi}\right)$,

$$
\left|\partial^{\beta}\left(T_{\hat{x}_{i}}^{p\left(x_{i}\right)} F-T_{\hat{x}}^{p(x)} F\right)(x)\right| \leq C(6 L)^{|\beta|+1} \underline{S}_{|\beta|}^{2 \xi} h_{\underline{s}^{2 \xi}}(3 L d(x)) .
$$

As in [16] we conclude (using [16, Proposition 4.9]) that there exist $W=W(p) \in \mathfrak{W}$ and $M=M(p)>0$ such that for all $i \in \mathbb{N}$, all $x \in \mathbb{R}^{n} \backslash E$ with $d(x)<r_{0} /\left(3 B_{1}\right)$, and all $\beta \in \mathbb{N}^{n}$,

$$
\left|\varphi_{i, p}^{(\beta)}(x)\right| \leq M W_{|\beta|} \Pi(p, x)
$$


where, by [16, Corollary 3.11],

$$
\begin{aligned}
\Pi(p, x) & =\exp \left(\frac{A_{1}(n)}{p} \sigma^{\star}\left(\frac{b_{1} p}{9 A_{2}(n)} d(x)\right)\right) \\
& \leq\left(\frac{e}{h_{\underline{s}^{\eta}}\left(\frac{b_{1} p d(x)}{9 A_{2}(n) B}\right)}\right)^{\frac{A_{1}(n) B}{p}}, \quad \text { for some } B \geq 1 \text { and all } \eta>0 .
\end{aligned}
$$

( $b_{1}$ is the universal constant from [16, Lemma 4.7] and $A_{1}(n) \leq A_{2}(n)$ are constants depending only on $n$.) By (11), we may assume that $\underline{S}^{2 \xi} \leq W$. Then, by (21), (22), (23), and [16. Lemma 4.7], for $x \in \mathbb{R}^{n} \backslash E$ with $d(x)<\min \left\{r_{0} /\left(3 B_{1}\right), 1 /\left(3 L \underline{s}_{1}^{2 \xi}\right)\right\}$,

$$
\begin{aligned}
& \left|\partial^{\alpha}\left(f-T_{\hat{x}}^{p(x)} F\right)(x)\right| \\
& \quad \leq \sum_{\beta \leq \alpha} \frac{\alpha !}{\beta !(\alpha-\beta) !} \cdot 12^{2 n} \cdot M W_{|\alpha|-|\beta|} \Pi(p, x) \cdot C(6 L)^{|\beta|+1} \underline{S}_{|\beta|}^{2 \xi} h_{\underline{s}^{2 \xi}}(3 L d(x)) \\
& \quad \leq 12^{2 n} C M\left(\sum_{j=0}^{|\alpha|} \frac{|\alpha| ! n^{|\alpha|+j}}{j !(|\alpha|-j) !}(6 L)^{j+1} W_{|\alpha|-j} \underline{S}_{j}^{2 \xi}\right) \Pi(p, x) h_{\underline{s}^{2 \xi}}(3 L d(x)) \\
& \quad \leq 6 \cdot 12^{2 n} L C M n^{|\alpha|} W_{|\alpha|}\left(\sum_{j=0}^{|\alpha|} \frac{|\alpha| !}{j !(|\alpha|-j) !}(6 L n)^{j}\right) \Pi(p, x) h_{\underline{s}^{2 \xi}}(3 L d(x)) \\
& \quad=6 \cdot 12^{2 n} L C M(n(1+6 L n))^{|\alpha|} W_{|\alpha|} \Pi(p, x) h_{\underline{s}^{2 \xi}}(3 L d(x)) .
\end{aligned}
$$

By Proposition 3, there is $H \geq 1$ (independent of $\xi$ ) such that $h_{\underline{s}^{2 \xi}}(t) \leq h_{\underline{s}^{4 \xi}}(H t)^{2}$ for $t>0$. Let us choose $L$ according to (19) and such that $p:=2 \overline{7} A_{2}(n) B \bar{H} L / b_{1} \geq$ $A_{1}(n) B$ is an integer. Then, by (24) and since $h_{\underline{s}^{4 \xi}} \leq 1$,

$$
\Pi(p, x) h_{\underline{s^{2}}}(3 L d(x)) \leq \frac{e h_{\underline{s}^{2 \xi}}(3 L d(x))}{h_{\underline{s}^{4} \xi}(3 H L d(x))} \leq e h_{\underline{s}^{4 \xi}}(3 H L d(x))
$$

and we obtain (20). (Note that $M$ depends on $p$, and hence on $L$, which results in the non-explicit dependence of $M_{1}$.)

- Let us finish the proof of Theorem 1, By (15) and (20), for all $x \in \mathbb{R}^{n} \backslash E$ with $d(x)<\min \left\{r_{0} /\left(3 B_{1}\right), 1 /\left(3 L \underline{s}_{1}^{2 \xi}\right)\right\}$ and all $\alpha \in \mathbb{N}^{n}$,

$$
\left|f^{(\alpha)}(x)\right| \leq\left|\left(T_{\hat{x}}^{p(x)} F\right)^{(\alpha)}(x)\right|+\left|\partial^{\alpha}\left(f-T_{\hat{x}}^{p(x)} F\right)(x)\right| \leq C M^{|\alpha|+1} W_{|\alpha|}
$$

for a suitable constant $M=M(n, \omega, L)$.

Let us fix a point $a \in E$ and $\alpha \in \mathbb{N}^{n}$. Since $\Gamma_{s^{2 \xi}}(t) \rightarrow \infty$ as $t \rightarrow 0$, we have $|\alpha|<p(x)$ if $x \in \mathbb{R}^{n} \backslash E$ is sufficiently close to $a$. Thus, as $x \rightarrow a$,

$$
\begin{aligned}
& \left|f^{(\alpha)}(x)-F^{\alpha}(a)\right| \\
& \leq\left|\partial^{\alpha}\left(f-T_{\hat{x}}^{p(x)} F\right)(x)\right|+\left|\left(T_{\hat{x}}^{p(x)} F\right)^{(\alpha)}(x)-F^{\alpha}(\hat{x})\right|+\left|F^{\alpha}(\hat{x})-F^{\alpha}(a)\right| \\
& =O\left(h_{\underline{s}^{4} \xi}\left(K_{3} L d(x)\right)\right)+O(d(x))+O(|\hat{x}-a|),
\end{aligned}
$$

by (13), (16), and (20). Hence $f^{(\alpha)}(x) \rightarrow F^{\alpha}(a)$ as $x \rightarrow a$. We may conclude that $f \in C^{\infty}\left(\mathbb{R}^{n}\right)$ and extends $F$. After multiplication with a suitable cut-off function of class $\mathcal{B}^{\{\omega\}}$ with support in $\left\{x: d(x)<\min \left\{r_{0} /\left(3 B_{1}\right), 1 /\left(3 L \underline{s}_{1}^{2 \xi}\right)\right\}\right\}$, we find that $f \in \mathcal{B}^{\{\omega\}}\left(\mathbb{R}^{n}\right)$ thanks to (12), (25), and [16, Lemma 2.4(5)]. The proof of Theorem1 is complete. 


\section{Conchye, good, And strong Weight functions}

In [16, Definition 3.5] we called a weight function $\sigma$ good if its associated weight matrix $\mathfrak{S}$ satisfies (1). A non-quasianalytic weight function $\omega$ is called strong if there is a constant $C>0$ such that

$$
\int_{1}^{\infty} \frac{\omega(t u)}{u^{2}} d u \leq C \omega(t)+C, \quad \text { for all } t>0 .
$$

Otherwise put, $\omega$ is strong if and only if it is equivalent to the concave weight function $\kappa=\kappa(\omega)$ defined in (10). In [16] we asked the following questions:

Question 3.21: Is every concave weight function equivalent to a good one?

Question 5.11: Is every strong weight function equivalent to a good one?

We will give partial answers to these questions and reveal some related connections in Theorem 11 below.

In [16] it was important that the associated weight matrix itself satisfies (1) as explained after the proof of Proposition 3. Since we could overcome this problem (by introducing $\mathfrak{V}=\left\{V^{\xi}\right\}$ ), it is more natural to allow for a wider concept of goodness. For completeness we will also treat the Beurling case. A weight function $\omega$ is called $R$-good if there exists a weight matrix $\mathfrak{M}$ satisfying

$$
\forall M \in \mathfrak{M} \exists N \in \mathfrak{M} \exists C \geq 1 \forall 1 \leq j \leq k: \frac{\mu_{j}}{j} \leq C \frac{\nu_{k}}{k}
$$

such that $\mathcal{B}^{\{\omega\}}=\mathcal{B}^{\{\mathfrak{M}\}}$. Recall that $\mu_{k}:=M_{k} / M_{k-1}$ and $\nu_{k}:=N_{k} / N_{k-1}$. Similarly, $\omega$ is called $B$-good if there exists a weight matrix $\mathfrak{M}$ satisfying

$$
\forall N \in \mathfrak{M} \exists M \in \mathfrak{M} \exists C \geq 1 \forall 1 \leq j \leq k: \frac{\mu_{j}}{j} \leq C \frac{\nu_{k}}{k}
$$

such that $\mathcal{B}^{(\omega)}=\mathcal{B}^{(\mathfrak{M})}$.

The next lemma, which is inspired by [5, Proposition 4.15], implies that for any weight matrix $\mathfrak{M}$ satisfying (26) (resp. (27)) there is a weight matrix $\mathfrak{S}$ consisting of strongly log-convex weight sequences such that $\mathcal{B}^{\{\mathfrak{M}\}}=\mathcal{B}^{\{\mathfrak{S}\}}\left(\operatorname{resp} . \mathcal{B}^{(\mathfrak{M})}=\mathcal{B}^{(\mathfrak{S})}\right)$.

Lemma 8. Assume that $1=\mu_{0} \leq \mu_{1} \leq \cdots$ and $1=\nu_{0} \leq \nu_{1} \leq \cdots$ satisfy

$$
\exists C>0: \frac{\mu_{j}}{j} \leq C \frac{\nu_{k}}{k}, \quad \text { for all } j \leq k .
$$

Then the sequence $\tilde{\nu}$ defined by

$$
\frac{\tilde{\nu}_{k}}{k}:=\inf _{\ell \geq k} \frac{\nu_{\ell}}{\ell}, \quad \tilde{\nu}_{0}:=1,
$$

is such that $\tilde{\nu}_{k} / k$ is increasing and $C^{-1} \mu \leq \tilde{\nu} \leq \nu$.

The next two corollaries are immediate from Lemma 8 and results of [12, [13, and [14].

Corollary 9. Let $\mathfrak{M}$ be a weight matrix with the property that for all $M \in \mathfrak{M}$ there is $N \in \mathfrak{M}$ such that $\left(M_{k+1} / N_{k}\right)^{1 / k}$ is bounded. Consider the following conditions:

(a) M satisfies (26).

(b) There is a weight matrix $\mathfrak{S}$ consisting of strongly log-convex weight sequences such that $\mathcal{B}^{\{\mathfrak{M}\}}=\mathcal{B}^{\{\mathfrak{S}\}}$.

(c) $\mathcal{B}^{\{\mathfrak{M}\}}$ is stable under composition.

(d) $\forall M \in \mathfrak{M} \exists N \in \mathfrak{M} \exists C>0 \forall j \leq k: m_{j}^{1 / j} \leq C n_{k}^{1 / k}$. 
Then $(\mathrm{a}) \Leftrightarrow(\mathrm{b}) \Rightarrow(\mathrm{c}) \Leftrightarrow(\mathrm{d})$. If additionally $\mathfrak{M}$ satisfies

$$
\forall M \in \mathfrak{M} \exists N \in \mathfrak{M}: \mu_{k} \lesssim N_{k}^{1 / k},
$$

then all four conditions are equivalent.

Corollary 10. Let $\mathfrak{M}$ be a weight matrix with the property that for all $N \in \mathfrak{M}$ there is $M \in \mathfrak{M}$ such that $\left(M_{k+1} / N_{k}\right)^{1 / k}$ is bounded. Consider the following conditions:

(a) $\mathfrak{M}$ satisfies (27).

(b) There is a weight matrix $\mathfrak{S}$ consisting of strongly log-convex weight sequences such that $\mathcal{B}^{(\mathfrak{M})}=\mathcal{B}^{(\mathfrak{S})}$.

(c) $\mathcal{B}^{(\mathfrak{M})}$ is stable under composition.

(d) $\forall N \in \mathfrak{M} \exists M \in \mathfrak{M} \exists C>0 \forall j \leq k: m_{j}^{1 / j} \leq C n_{k}^{1 / k}$.

Then $(\mathrm{a}) \Leftrightarrow(\mathrm{b}) \Rightarrow(\mathrm{c}) \Leftrightarrow(\mathrm{d})$. If additionally $\mathfrak{M}$ satisfies

$$
\forall N \in \mathfrak{M} \exists M \in \mathfrak{M}: \mu_{k} \lesssim N_{k}^{1 / k},
$$

then all four conditions are equivalent.

In general, $(\mathrm{c}) \nRightarrow(\mathrm{b})$ in neither of the corollaries which follows from [12, Example 3.6]. Note that if $M=N$ then (28) and (29) reduce to a condition which is usually called moderate growth or $M$.

For weight functions $\omega$ we get a full characterization.

Theorem 11. Let $\omega$ be a weight function satisfying $\omega(t)=o(t)$ as $t \rightarrow \infty$. Then the following are equivalent.

(a) $\omega$ is equivalent to a concave weight function.

(b) $\exists C>0 \exists t_{0}>0 \forall \lambda \geq 1 \forall t \geq t_{0}: \omega(\lambda t) \leq C \lambda \omega(t)$.

(c) $\mathcal{B}^{\{\omega\}}$ is stable under composition.

(d) $\mathcal{B}^{(\omega)}$ is stable under composition.

(e) There is a weight matrix $\mathfrak{S}$ consisting of strongly log-convex weight sequences such that $\mathcal{B}^{\{\omega\}}=\mathcal{B}^{\{\mathfrak{S}\}}$.

(f) There is a weight matrix $\mathfrak{S}$ consisting of strongly log-convex weight sequences such that $\mathcal{B}^{(\omega)}=\mathcal{B}^{(\mathfrak{S})}$.

(g) $\omega$ is R-good.

(h) $\omega$ is B-good.

Notice that the conditions in the theorem are furthermore equivalent to the classes $\mathcal{B}^{\{\omega\}}$ and $\mathcal{B}^{(\omega)}$ to be stable under inverse/implicit functions and solving ODEs, and, in terms of the associated weight matrix $\mathfrak{W}=\left\{W^{\xi}\right\}_{\xi>0}$, to

$$
\forall \xi>0 \exists \eta>0:\left(w_{j}^{\xi}\right)^{1 / j} \leq C\left(w_{k}^{\eta}\right)^{1 / k} \quad \text { for } j \leq k,
$$

as well as

$$
\forall \eta>0 \exists \xi>0:\left(w_{j}^{\xi}\right)^{1 / j} \leq C\left(w_{k}^{\eta}\right)^{1 / k} \quad \text { for } j \leq k,
$$

see 13. In the forthcoming paper [4] we shall see that they are also equivalent to the property that $\mathcal{B}^{\{\omega\}}$, resp. $\mathcal{B}^{(\omega)}$, can be described by almost analytic extensions; see also [11.

Proof. The equivalence of the first four conditions (a)-(d) is well-known, see e.g. [13, which is based on [10, Lemma 1] and [3. That (a) implies (e) and (f) follows from Proposition 3. (e) $\Rightarrow$ (c) and (f) $\Rightarrow$ (d) are clear; cf. 12. The equivalences (e) $\Leftrightarrow$ (g) and (f) $\Leftrightarrow$ (h) follow from Lemma 8 . 


\section{Appendix A. Weight matrices}

By a weight matrix we mean a family $\mathfrak{M}$ of weight sequences $M \geq(k !)_{k}$ which is totally ordered with respect to the pointwise order relation on sequences, i.e.,

- $\mathfrak{M} \subseteq \mathbb{R}^{\mathbb{N}}$,

- each $M \in \mathfrak{M}$ is a weight sequence, which means that $M_{0}=1, M_{k}^{1 / k} \rightarrow \infty$, and $M$ is log-convex,

- each $M \in \mathfrak{M}$ satisfies $k ! \leq M_{k}$ for all $k$,

- for all $M, N \in \mathfrak{M}$ we have $M \leq N$ or $M \geq N$.

For a weight matrix $\mathfrak{M}$ and an open $U \subseteq \mathbb{R}^{n}$ we consider the Roumieu class

$$
\mathcal{B}^{\{\mathfrak{M}\}}(U):=\operatorname{ind}_{M \in \mathfrak{M}} \mathcal{B}^{\{M\}}(U),
$$

and the Beurling class

$$
\mathcal{B}^{(\mathfrak{M})}(U):=\operatorname{proj}_{M \in \mathfrak{M}} \mathcal{B}^{(M)}(U) .
$$

For weight matrices $\mathfrak{M}, \mathfrak{N}$ we have (cf. [12])

$$
\begin{aligned}
& \mathcal{B}^{\{\mathfrak{M}\}} \subseteq \mathcal{B}^{\{\mathfrak{N}\}} \Leftrightarrow \quad \forall M \in \mathfrak{M} \exists N \in \mathfrak{N}:\left(M_{k} / N_{k}\right)^{1 / k} \text { is bounded, } \\
& \mathcal{B}^{(\mathfrak{M})} \subseteq \mathcal{B}^{(\mathfrak{N})} \quad \Leftrightarrow \quad \forall N \in \mathfrak{N} \exists M \in \mathfrak{M}:\left(M_{k} / N_{k}\right)^{1 / k} \text { is bounded. }
\end{aligned}
$$

Analogous equivalences hold for the local classes

$$
\mathcal{E}^{\{\mathfrak{M}\}}(U):=\operatorname{proj}_{V \Subset U} \mathcal{B}^{\{\mathfrak{M}\}}(V) \quad \text { and } \quad \mathcal{E}^{(\mathfrak{M})}(U):=\operatorname{proj}_{V \Subset U} \mathcal{B}^{(\mathfrak{M})}(V) .
$$

\section{REFERENCES}

[1] J. Bonet, R. W. Braun, R. Meise, and B. A. Taylor, Whitney's extension theorem for nonquasianalytic classes of ultradifferentiable functions, Studia Math. 99 (1991), no. 2, 155-184.

[2] J. Bonet, R. Meise, and B. A. Taylor, On the range of the Borel map for classes of nonquasianalytic functions, Progress in functional analysis (Peñiscola, 1990), North-Holland Math. Stud., vol. 170, North-Holland, Amsterdam, 1992, pp. 97-111. MR 1150740

[3] C. Fernández and A. Galbis, Superposition in classes of ultradifferentiable functions, Publ. Res. Inst. Math. Sci. 42 (2006), no. 2, 399-419.

[4] S. Fürdös, D. N. Nenning, A. Rainer, and G. Schindl, Almost analytic extensions of ultradifferentiable functions, in preparation.

[5] J. Jiménez-Garrido and J. Sanz, Strongly regular sequences and proximate orders, Journal of Mathematical Analysis and Applications 438 (2016), no. 2, 920-945.

[6] J. Jiménez-Garrido, J. Sanz, and G. Schindl, Sectorial extensions, via Laplace transforms, in ultraholomorphic classes defined by weight functions, (2017), arXiv:1710.10081.

[7] H. Komatsu, Ultradistributions. I. Structure theorems and a characterization, J. Fac. Sci. Univ. Tokyo Sect. IA Math. 20 (1973), 25-105.

[8] W. Matsumoto, Characterization of the separativity of ultradifferentiable classes, J. Math. Kyoto Univ. 24 (1984), no. 4, 667-678.

[9] R. Meise and B. A. Taylor, Whitney's extension theorem for ultradifferentiable functions of Beurling type, Ark. Mat. 26 (1988), no. 2, 265-287.

[10] J. Peetre, Concave majorants of positive functions, Acta Math. Acad. Sci. Hungar. 21 (1970), 327-333.

[11] H.-J. Petzsche and D. Vogt, Almost analytic extension of ultradifferentiable functions and the boundary values of holomorphic functions, Math. Ann. 267 (1984), no. 1, 17-35.

[12] A. Rainer and G. Schindl, Composition in ultradifferentiable classes, Studia Math. 224 (2014), no. 2, 97-131.

[13] _ Equivalence of stability properties for ultradifferentiable function classes, Rev. R. Acad. Cienc. Exactas Fis. Nat. Ser. A Math. RACSAM. 110 (2016), no. 1, 17-32.

[14] _ Extension of Whitney jets of controlled growth, Math. Nachr. 290 (2017), no. 14-15, 2356-2374, doi:10.1002/mana.201600321. 
[15] — On the Borel mapping in the quasianalytic setting, Math. Scand. 121 (2017), 293310.

[16] A. Rainer and G. Schindl, On the extension of Whitney ultrajets, Studia Math. 245 (2019), no. 3, 255-287, doi:10.4064/sm170906-23-11.

[17] G. Schindl, Exponential laws for classes of Denjoy-Carleman differentiable mappings, Ph.D. thesis, Universität Wien, 2014, http://othes.univie.ac.at/32755/1/2014-01-26_0304518.pdf.

[18] - Characterization of ultradifferentiable test functions defined by weight matrices in terms of their Fourier transform, Note di Matematica 36 (2016), no. 2, 1-35, doi:10.1285/i15900932v36n2p1.

A. Rainer: Fakultät Für Mathematik, Universität Wien, Oskar-MorgensternPlatz 1, A-1090 Wien, Austria

E-mail address: armin.rainer@univie.ac.at

G. Schindl: Departamento de Álgebra, Análisis Matemático, Geometría y Topología, Facultad de Ciencias, Universidad de Valladolid, Paseo de Belén 7, 47011 Valladolid, SPAIN

E-mail address: gerhard.schindl@univie.ac.at 